\title{
Zwischen Sicherung und Gestaltung - Varianten mitbestimmter Innovation in der Industrie
}

In den aktuellen Debatten zur Innovationsfähigkeit von Unternehmen werden betriebliche Interessenvertretung und Unternehmensmitbestimmung, zumindest im Hinblick auf Produktinnovationen, als verzögernde oder gar blockierende Faktoren angesehen. Ob und wie Mitbestimmung einen Beitrag zur Bearbeitung des Innovations-Dilemmas in der Forschung und Entwicklung leisten kann, gerät seltener in den Blick. Ausgehend von empirischen Befunden werden Konstellationen, Akteure und Orientierungen benannt, die deutlich machen, welche Rolle Mitbestimmung bei der Sicherung und Gestaltung von technischen Innovationsprozessen spielen kann. Fälle von „good practices“ innovationsförderlicher Mitbestimmung bleiben freilich voraussetzungs- und anspruchsvoll.

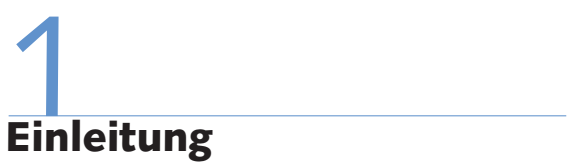

Die sozialwissenschaftliche Innovationsforschung macht seit geraumer Zeit deutlich, dass es sich bei der Suche nach und der Generierung von Innovationen um ein höchst anspruchsvolles und fragiles, weil ungewisses Unterfangen handelt. In einer begründeten Absetzung von deterministischen und linearen Interpretationen und Konzeptualisierungen von Innovationsverläufen wurde in neueren theoretischen wie empirischen Beiträgen (im Überblick: Fagerberg et al. 2005; Blättel-Mink 2006) das Innovations-Paradox oder Innovations-Dilemma herausgearbeitet und benannt. Im Kern geht es um das Problem, dass man schwerlich nach etwas suchen kann, was man noch gar nicht kennt. Das aber heißt: $\mathrm{Ob}$ und wie Innovationen tatsächlich erfolgen und erfolgreich sein werden, ist nicht ex ante kalkulierbar. Sowohl die Wahrscheinlichkeit von Innovationen als auch deren mögliches Risiko schälen sich aufgrund unberechenbarer sachlicher, zeitlicher und sozialer Ungewissheiten erst im Prozess des „making innovation" heraus. Betriebliche Akteure, die im Rahmen von Innovationsprozessen arbeiten und Entscheidungen treffen, tun dies also unter enormen Unsicherheiten. Was gleichwohl wiederum gerade nicht heißt, dass Innovationen in gänzlich unreglementierten, „freien “ organisatorischen und sozialen Räumen stattfinden.

Aus der Sicht des Managements von Unternehmen hat Werner Rammert das In-
novations-Dilemma anschaulich benannt: „Unterwerfen sie (die Firmen) die Wissenschaftler und ihr innovatives Handeln zu sehr den üblichen Routinen betrieblicher Kontrolle und wirtschaftlichen Kalküls, dann laufen sie Gefahr, die erwünschte Kreativität im Keim zu ersticken und das anvisierte innovative Potenzial kleinlich einzuzäumen; ließe man umgekehrt der Forschung freien Lauf und verzichtete auf Zwischenziele und jegliche Anwendungsorientierung, finanzierte man zwar eine tolle Truppe origineller Denker und Forscher, aber ohne absehbaren Gewinn" (Rammert 2008, S. 312). Welche Ressourcen wie mobilisiert und organisiert werden, in welchen Organisationsformen, mit welchen Kompetenzen, ist für Innovationsverläufe und deren Ergebnisse deshalb von erheblicher Bedeutung.

Erst durch genaue Rekonstruktion und empirisch begründete Evaluation von Innovationsprojekten lassen sich Aufschlüsse darüber gewinnen, wie ein zugleich innovationsförderlicher und geregelter Umgang mit Ungewissheit erfolgt und ermöglicht werden kann. Es fällt auf, dass Aspekten der Mitbestimmung in diesem Zusammenhang von der sozialwissenschaftlichen Innovationsforschung bislang nur eine geringe Aufmerksamkeit gewidmet wurde. Entsprechende Stichworte sucht man in den Sachregistern der einschlägigen Literatur zumeist vergeblich, nur wenige empirische Studien greifen dieses Thema auf. Die in der jüngeren Zeit neubelebte Rezeption von Joseph Schumpeter als bedeutendem Innovations-Theoretiker dürfte dabei auch eine Rolle spielen. Denn die vorherrschende Konzentration auf den dynamischen
Entrepreneur als Innovationsgenerator hat sehr stark Dimensionen der unternehmerischen Führungspersönlichkeit betont und die „schöpferische Zerstörung“ in den neoliberalen Diskurs eingepasst. Weniger zum Tragen kam demgegenüber der Umstand, dass Innovationen überwiegend in kooperativen Zusammenhängen stattfinden, die in großbetrieblichen Strukturen angesiedelt sind. Schumpeter selbst hatte in seinen späteren Arbeiten thematisiert, dass die Unternehmerfunktion durch Bürokratisierung und Automatisierung zunehmend obsolet werde (Fagerberg 2005), wodurch Muster „schöpferischer Zerstörung“ abgelöst würden durch Muster einer „schöpferischen Akkumulation" von Wissen und Innovationsressourcen (Breschi et al.2000). Es geht hier also meist nicht um schlagartige Erweiterungen technologischer Möglichkeiten, mit denen zuvor nicht innovierende Unternehmen plötzlich zu den wichtigsten Innovationsträgern aufsteigen würden, sondern vor allem um Formen der „Vertiefung“ innerhalb eines wohl konturierten Spektrums mit relativ hoher Kontinuität und Stabilität bei der Innova-

\footnotetext{
Hans Joachim Sperling, Dr., Soziologe, Mitarbeiter am Soziologischen Forschungsinstitut (SOFI) an der Universität Göttingen Arbeitsschwerpunkte: Industrie- und Arbeitssoziologie, Industrielle Beziehungen. e-mail: joachim.sperling@sofi.unigoettingen.de Harald Wolf, PD Dr., Soziologe, Mitarbeiter am SOFI. Arbeitsschwerpunkte: Arbeitssoziologie, Gesellschaftstheorie. e-mail: harald.wolf@sofi.uni-goettingen.de
} 
tionsträgerschaft bzw. der Hierarchie der Innovateure.

Richtet man das Interesse auf die konkreten sozialen Bedingungen betrieblicher Innovationsprozesse und nimmt dabei insbesondere die Rolle der Interessenvertretung in den Blick, dann ist - schon wegen der Vielfalt möglicher betrieblicher „Innovations"-Phänomene und -Schauplätze eine bewusste Begrenzung des empirischen Zugriffs geboten. Deshalb zielt hier unser Fokus nur auf jenen Ausschnitt von Neuerungen, den die Organisation for Economic Co-operation and Development (OECD) in ihrem häufig zitierten „Oslo Manual“ als „technologische Produktinnovationen“ definiert: „Technological product (...) innovations comprise implemented technologically new products (...) and significant technological improvements in products (...). A [technological product] innovation has been implemented if it has been introduced on the market (...)" (OECD 1995, S. 31). Die betriebliche Realisierung so gefasster technischer Produktinnovationen ist ein komplexer Prozess, der eine ganze Reihe von Aktivitäten einschließt. Der abstrakt bestimmte Kanon von Aktivitäten, den die OECD hierfür als einschlägig erachtet, reicht von der Wissensgenerierung bzw. -beschaffung über die Entwicklung und Konstruktion bis hin zur Implementation in die Fertigung (vgl. OECD 1995, S. 39ff.).

Im Folgenden wollen wir mit unserem Beitrag unterschiedliche Konstellationen, Orientierungen und Aushandlungsformen im Spannungsfeld von Innovationsaktivitäten und Mitbestimmung herausarbeiten. Dies geschieht auf Grundlage von Zwischenergebnissen eines Forschungsprojektes zum Thema „Innovation und Mitbestimmung: Regulierungsbedarfe, Interessenorientierungen und die Entwicklung von Spielregeln für Innovationshandeln“, das am Soziologischen Forschungsinstitut (SOFI) an der Universität Göttingen durchgeführt wird. ${ }^{1}$ Zunächst skizzieren wir die Fragestellungen dieses Projektes und die empirische Grundlage unserer Ausführungen (Abschnitt 2). In Abschnitt 3 wird auf Umstrukturierungen und den wachsenden Ökonomisierungsdruck auf die Forschungs- und Entwicklungsbereiche in den Untersuchungsbetrieben eingegangen - Entwicklungen, die Regelungsbedarf erzeugen und an Betriebsräte neue Anforderungen stellen. Anschließend werden verschiedene Varianten von „mitbestimm- ter Innovation", die sich zwischen Sicherungs- und Gestaltungsfunktionen bewegen, dargestellt (Abschnitt 4). Resümierend weisen wir schließlich noch auf einige Bedingungen variierender Mitbestimmungsformen bei Innovationsprozessen hin (Abschnitt 5) und markieren (auch an einem Beispiel) die Grenzen ihrer Gestaltung durch betriebliche Interessenvertretung - auch im Fall von,good practices“ (Abschnitt 6). Abschnitt 7 zieht ein knappes Fazit.

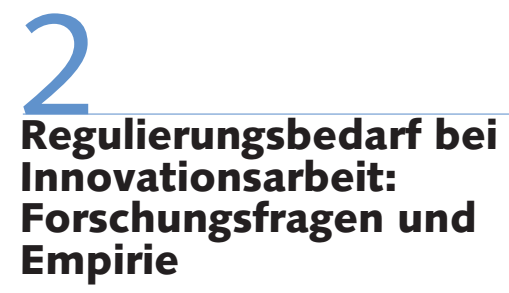

Wir gehen davon aus, dass für betriebliche Innovationen Problemkonstellationen charakteristisch sind, die in der vorherrschenden Diskussion über Innovationen unterbelichtet bleiben. Probleme ergeben sich vor allem daraus, dass die Organisation von Innovationsprozessen auf spezifische Ressourcen angewiesen ist (individuelle wie organisationale Wissensbestände und Innovationskompetenzen, Kooperationsstrukturen), die langfristig aufgebaut und gepflegt werden müssen. Betriebliche Innovationsstrategien lassen sich nur dann und in dem Maße verwirklichen, wie sie auf die Kooperationsbereitschaft und Kooperationsfähigkeit von entsprechend qualifizierten Beschäftigten zurückgreifen können. Für die Innovationskraft von Unternehmen zentral sind zudem die Erwartungen, welche die mit Innovation befassten Beschäftigten an ihre Tätigkeit und an ihre Arbeits- und Lebensbedingungen haben, sowie die Möglichkeit, diese mit den betrieblichen Anforderungen in eine akzeptable, möglichst sogar attraktive Balance zu bringen.

Wenn Organisationen die Nutzung, den Erhalt und die Entwicklung der angesprochenen Ressourcen für Innovationsprozesse nachhaltig sichern wollen, ergeben sich spezifische Regulierungsbedarfe. Diese entstehen

(1) aus dem Charakter von Innovationsarbeit als Projektarbeit (etwa die Regelung von Arbeitszeiten sowie die Regelung des Umgangs mit Veränderungen von Zielsetzungen und Rahmenbedingungen von Projekten);
(2) aus dem Erfordernis, einen funktionsbzw. bereichsübergreifenden Wissenstransfer zu gewährleisten (beispielsweise die Regelung funktions- bzw. bereichsübergreifender Kooperation sowie die Sicherstellung des Einbezugs „impliziten Wissens");

(3) aus den Konditionen der Nutzung von Innovationskompetenzen (Sicherstellung von Partizipation der Beschäftigten an Innovationsaufgaben, Schaffung einer innovationsförderlichen Arbeitsorganisation);

(4) und schließlich aus dem Ziel, Innovationskompetenzen zu erhalten und weiterzuentwickeln (Begrenzung der Anforderungen und Belastungen, Gewährleistung der Lernförderlichkeit der Arbeitsorganisation und von adäquater Weiterbildung).

Für diese Regulierungsbedarfe ist charakteristisch, so die Annahme, dass sie den Horizont der Selbstregulation von hochqualifizierten Innovationsarbeitern und Innovationsarbeiterinnen im Rahmen von Projekten oder "Communities of Practice“, so konstitutiv diese für Innovationen auch sind, übersteigen. Es scheint vielmehr nötig, den Erfordernissen angepasste allgemeine und für alle Beteiligten akzeptable und gesicherte Spielregeln für Innovationshandeln zu etablieren. Damit sind in dieser Frage auch die Akteure betrieblicher Mitbestimmung in ihrer Gestaltungskompetenz gefordert, angesichts der prinzipiellen Unsicherheiten und Ungewissheiten von Produktinnovationen einen Part zu spielen. $\mathrm{Ob}$ und wie Mitbestimmung diese Anforderungen aufzugreifen und zu verarbeiten in der Lage ist: vor allem dieser Teilfrage versucht der Artikel nachzugehen. Kann das Regulierungssystem der Mitbestimmung auf den Ressourceneinsatz und die Organisations- und Arbeitsbedingungen bei technischen Innovationen in Unternehmen einwirken, in welchen Konstellationen, mit Blick auf welche Akteure, und wie gestalten sich dabei die Spielregeln der Innovation?

Die empirische Grundlage der folgenden Ausführungen sind vier Intensiv-Fallstudien, die vom SOFI im Rahmen des erwähnten qualitativ-explorativen For-

\footnotetext{
1 Die Forschungsarbeiten werden von der HansBöckler-Stiftung finanziert. Zum SOFI-Projektteam gehören neben den beiden Autoren Jürgen Kädtler und Volker Wittke.
} 


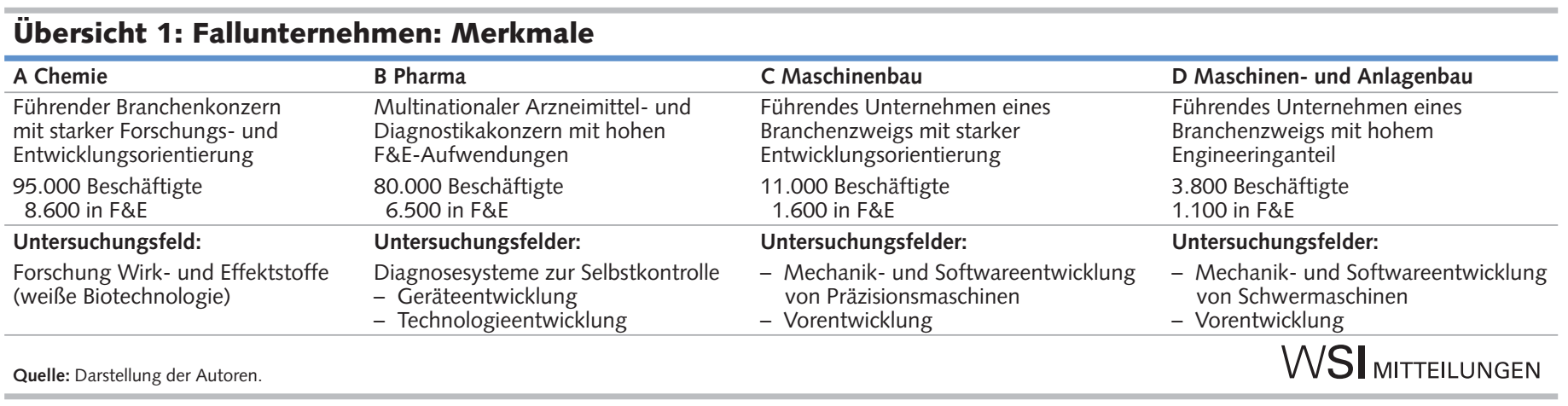

schungsprojektes in den Jahren 2008 und 2009 durchgeführt wurden. Bei den vier Unternehmensfällen handelt es sich um jeweils zwei große Unternehmen der Chemie- und Pharmaindustrie sowie zwei Unternehmen des Maschinen- und des Anlagenbaus. In den vier Unternehmen wurden insgesamt 109 Gespräche geführt, davon 33 Expertengespräche mit Managementvertretern und Interessenvertretern und 76 Beschäftigteninterviews mit Naturwissenschaftlern, Ingenieuren, Technikern und Laboranten.

Ausschlaggebend für die Auswahl der Unternehmen war, dass es sich um Unternehmen handelt, in denen aufgrund der Verteilung der organisatorischen und personellen Kapazitäten und Ressourcen der Innovationsbereich - also im Kern der Bereich Forschung und Entwicklung (F\&E) mit vor- und nachgelagerten Bereichen einen hohen Stellenwert hat. Die aus dieser Perspektive relevanten Merkmale der vier Unternehmen stellt Übersicht 1 dar.

Bei den untersuchten Unternehmen handelt es sich um Bastionen in den Kernbereichen der deutschen Industrie, die sich zum einen durch eine starke Innovationsorientierung im Hochtechnologiebereich auszeichnen. Zum anderen weisen sie durchweg starke Mitbestimmungskulturen auf, mit tief verankerten Mitbestimmungsinstitutionen auf Betriebsebene (Betriebsrat) wie auf Unternehmensebene (Mitbestimmung im Aufsichtsrat), die relativ großen Einfluss auf die Beschäftigungsund Arbeitsbedingungen haben. Allerdings zeichnen sich die Unternehmen branchenspezifisch durch deutlich kontrastierende Mitbestimmungskulturen aus.

Die bisherige Mitbestimmungskultur in der Chemie- und Pharmaindustrie (Kädtler 2006) wurde maßgeblich durch eine von Gewerkschaft und Arbeitgeberverbänden gemeinsam getragene „Chemiepartnerschaft" bestimmt, deren Arrangements auf der Verallgemeinerung eines unterneh- mensbezogenen Kooperationsmodells beruhte, das in den Nachkriegsjahrzehnten in der Großchemie entstand und in dessen Rahmen beträchtliche materielle Erfolge der Arbeitnehmervertretungen als Gegenleistung für das kooperative Mittragen der Unternehmensstrategien erzielt wurden. In diesem Zusammenhang ist das Engagement von Gewerkschaft und Betriebsräten für Produktinnovation und die Stärkung von F\&E in erster Linie strategisch motiviert. Es reflektiert die Relevanz der Innovation für die Wirtschaftlichkeit der Branchen und als Folge davon für Arbeitsplatzsicherheit und Verteilungsspielräume. Die arbeitsbezogenen Interessen der eigenen Kernklientel betrifft ein solches Innovationsengagement insoweit nur mittelbar. (Produkt-)Innovationsbezogene Problemkomplexe wurden hier bisher allenfalls für einen Teil der Kernklientel von Betriebsräten und Gewerkschaft - nämlich die gut organisierten Laborantinnen und Laboranten in der Forschung - nur dann unmittelbar zum Vertretungsthema, wenn deren Arbeitszeit- und Arbeitsplatzinteressen tangiert waren.

Im Mittelpunkt der Mitbestimmungskultur in der Metallindustrie steht die Vertretung der Interessen einer relativ hoch organisierten, auch die Betriebsräte dominierenden Facharbeiterschaft in Mittel- und Großbetrieben. Das konkrete Engagement der organisierten Arbeitnehmervertretungen im Hinblick auf Innovationen bezog sich in diesem Rahmen bisher in erster Linie auf technisch-organisatorische Prozessinnovationen. Innovationsarbeit und Innovationsarbeiterinnen bzw. Innovationsarbeiter konnten hier vor allem insofern ins Spiel kommen, als die Kooperationsbezüge zwischen gewerkschaftlicher Kernklientel und Produktinnovation oft relativ eng und Übergänge zwischen Facharbeiter-, Techniker- und Ingenieurpositionen, etwa über ein Aufbaustudium, nach wie vor nicht selten sind. Auf dieser Grund- lage erwachsen zudem vielfältige informelle Interessenarrangements, die als eine Art Unterbau in die institutionalisierten Aushandlungsbeziehungen eingehen können.

Die untersuchten Unternehmen sind folglich Unternehmen mit stabilen Traditionsbezügen und starken branchenmäßig ausgeprägten Mitbestimmungskulturen. Zugleich sind es Unternehmen mit einer markanten Ausrichtung auf technische Produktinnovationen. Konkret freilich handelt es sich hier durchaus um unterschiedliche Konstellationen, in denen Produktinnovationen entwickelt und Entscheidungen über Innovations-Entwicklungen getroffen werden, sodass auch unterschiedliche Ressourcen- und Regulierungsbedarfe anzutreffen sind. Auch im Hinblick auf die Ressourcen und strategischen wie operativen Orientierungen der Interessenvertretungen weisen die Mitbestimmungsansprüche und -wirkungen verschiedene Ausprägungen auf, die sich nicht allein aufgrund der Branchenspezifik ergeben. Auf der Basis von Teilauswertungen der erhobenen Empirie thematisieren wir im Folgenden Aspekte des Spannungsverhältnisses von Innovation und Mitbestimmung, die vor allem die Handlungsbedingungen und -perspektiven der betrieblichen Interessenvertretungen betreffen.

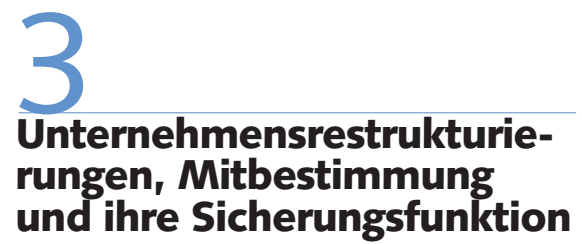

Für alle Unternehmen gilt, dass sie in den letzten Jahren mit gravierenden Marktveränderungen, Globalisierungstendenzen und Finanzmarktanforderungen konfrontiert wurden. Diese Herausforderungen prägen jeweils das strategische Handeln der Unternehmen wie auch die strategische Positionierung der Interessenvertretung. 


\begin{tabular}{|c|c|c|c|c|}
\hline & Mitbestimmungskultur & $\begin{array}{l}\text { Merkmale der } \\
\text { Innovationsprozesse }\end{array}$ & Art der Einflussnahme & Regelungsprozesse \\
\hline Fall B & Chemie-Sozialpartnerschaft & $\begin{array}{l}\text { Naturwissenschaftlich basierte } \\
\text { Wissenserzeugung im Rahmen } \\
\text { formalisierter Strukturen für die } \\
\text { Massenproduktion }\end{array}$ & $\begin{array}{l}\text { Sicherung von F\&E-Ressourcen } \\
\text { durch Kooperation mit Standort- } \\
\text { management; Betriebsrat als } \\
\text { Informationsquelle und Sprachrohr } \\
\text { auch für Innovationsarbeiter }\end{array}$ & $\begin{array}{l}\text { Neben Einbezug in allgemeine } \\
\text { Rahmenregelungen keine } \\
\text { konkreten Gestaltungsinitiativen } \\
\text { für Innovationsarbeit }\end{array}$ \\
\hline Fall C & Metall-Konfliktpartnerschaft & $\begin{array}{l}\text { Programmfertigung mit zeitlich } \\
\text { und sozial von der Produktion } \\
\text { abgekoppelten F\&E-Prozessen } \\
\text { auf ingenieurtechnischer Basis }\end{array}$ & $\begin{array}{l}\text { Sicherung von F\&E-Ressourcen } \\
\text { im Rahmen von Standortverein- } \\
\text { barungen; F\&E-ferne Betriebsrats- } \\
\text { arbeit }\end{array}$ & $\begin{array}{l}\text { Neben Einbezug in allgemeine } \\
\text { Rahmenregelungen keine } \\
\text { konkreten Gestaltungsinitiativen } \\
\text { für Innovationsarbeit }\end{array}$ \\
\hline
\end{tabular}

In allen Unternehmen sind die Akteure der Mitbestimmung aufgefordert gewesen, organisatorische Umstrukturierungen $\mathrm{zu}$ bewältigen und dabei auch Gefährdungen von Beschäftigung und von ganzen Arbeitsfeldern zum Gegenstand von Aushandlungen zu machen, allerdings auf unterschiedliche Weise und im Rahmen je spezifischer Handlungsbedingungen. Dabei geht es jeweils um die Sicherung der Produktionsbasis und der für Produktinnovationen relevanten F\&E-Sektoren.

So erweist sich das Chemieunternehmen $A$, nach einem nicht unerheblichen Personalabbau in den 1990er Jahren, seit einiger Zeit als prosperierend, was die Basis für eine stabile und wirksame Interessenvertretungsorganisation und -struktur ist. Damit kontrastiert das Pharmaunternehmen $B$, in dem die Organisationskraft der Interessenvertretung an Gewicht verloren hat, aufgrund eines Eigentümerwechsels von einem im Familienbesitz befindlichen Unternehmen zu einem börsennotierten Konzern mit Hauptsitz im europäischen Ausland und einer damit zunehmend auch auf den F\&E-Bereich durchschlagenden Marketing- und Shareholder-Value-Orientierung.

Auch die beiden Maschinenbauer kontrastieren deutlich. Das Unternehmen C erfuhr mit einem vor zehn Jahren erfolgten Börsengang ebenfalls einen tiefen Einschnitt in der Unternehmensentwicklung. Dies und der zugleich intensivierte Produktinnovations- und Kostenwettbewerb macht auch die Aufwendungen für Forschung und Entwicklung stärker begründungspflichtig. Dagegen wird von dem Unternehmen $D$, das aufgrund des Produktportfolios technologisch und ökonomisch mit stabileren Märkten zu tun hat, auch angesichts einer absehbaren Absatz-Schwächung auf den Ausbau und die Intensivierung der Entwicklung gesetzt. Dass es sich hier um ein weiterhin in Familienbesitz be- findliches Traditionsunternehmen handelt, ist hierfür ausschlaggebend.

In allen vier Unternehmen sind die Umstrukturierungen der letzten Jahre begleitet gewesen von Aushandlungsprozessen, in denen Management und betriebliche Interessenvertretung weitgehend einvernehmliche organisatorische und personelle Anpassungsmaßnahmen vereinbart haben, bei denen Konzessionen der Beschäftigten wie Arbeitszeitverlängerung eingebracht wurden, zumeist gegen die $\mathrm{Zu}$ sage von Beschäftigungssicherung. Waren es zwar in erster Linie die in Fertigung und Produktion beschäftigten Arbeitnehmer, die von den Sicherungszusagen im Hinblick auf Beschäftigung und Arbeitsbedingungen betroffen waren, so galten diese gleichwohl auch für die hochqualifizierten Beschäftigten, die in Bereichen von Forschung und Entwicklung tätig sind. Geht es für diese zwar vor allem auch um den besonderen Regelungsbedarf bei Innovationsarbeit, so haben doch die tief reichenden ökonomischen Veränderungen der letzten Jahre auch dieser Beschäftigtengruppe die allgemeine Schutz- und Regelungsbedürftigkeit von Arbeit und Beschäftigung verstärkt vor Augen geführt. Gerade dadurch - das zeigen die geführten Interviews durchweg, und das ist kein trivialer Befund, - hat sich für sie die Wichtigkeit der Sicherungsfunktionen von Betriebsrat und Mitbestimmung positiv bestätigt oder ist sogar aufgewertet worden: als Garanten eines auch in Umstrukturierungs- und Krisenzeiten noch relativ abgesicherten und regulierten Arbeits- und Beschäftigungsumfeldes.

Die bislang als ,sozialverträglich“"wahrgenommene Bewältigung von Einschnitten hat einerseits die Regelungsfähigkeit institutionalisierter Interessenvertretung auch für hochqualifizierte Arbeitskräfte im Innovationsbereich erwiesen, andererseits zugleich den stärker werdenden Druck ver- deutlicht, der durch Ökonomisierungstendenzen und enger gefasste Steuerung und Kontrolle auch auf sie ausgeübt wird womit neuer Regelungsbedarf speziell für Forschung und Entwicklung entsteht.

\section{4 \\ Varianten mitbestimmter Innovation}

Im Rahmen der mittlerweile zur Routine gewordenen Standortsicherungsvereinbarungen, aber auch darüber hinaus, finden in allen Unternehmen spezifischere Klärungen und Aushandlungen darüber statt, welcher Stellenwert dem Innovationsbereich im strategischen Konzept der Unternehmen zukommt und welche organisatorischen und personellen Ausstattungen der jeweiligen Forschungs- und Entwicklungseinheiten damit verbunden sein sollen. Art, Stoßrichtung und Reichweite der formellen wie informellen Aktivitäten, Absprachen oder Vereinbarungen, die in diesem Zusammenhang zu beobachten sind, weisen ein weites Spektrum auf. Zum Teil sind sie eher als begleitend und absichernd $\mathrm{zu}$ charakterisieren, insofern sie sich auf der Ebene der Setzung und Kontrolle von Rahmenbedingungen der Beschäftigungs- und Arbeitsverhältnisse auch für die Innovationsbereiche bewegen. Zum Teil finden wir die Betriebräte darüber hinaus aber auch in einer die Innovation und die Innovationsarbeit gezielt mitgestaltenden und insofern pro-aktiven Rolle.

\subsection{ZWISCHEN SICHERUNGS- FUNKTIONEN...}

Weitgehend auf Begleitung und Sicherung von Innovation beschränkt sich Mitbestimmung in den Fällen B und C (Übersicht 2). Im Pharmaunternehmen $B$ versucht der Be- 


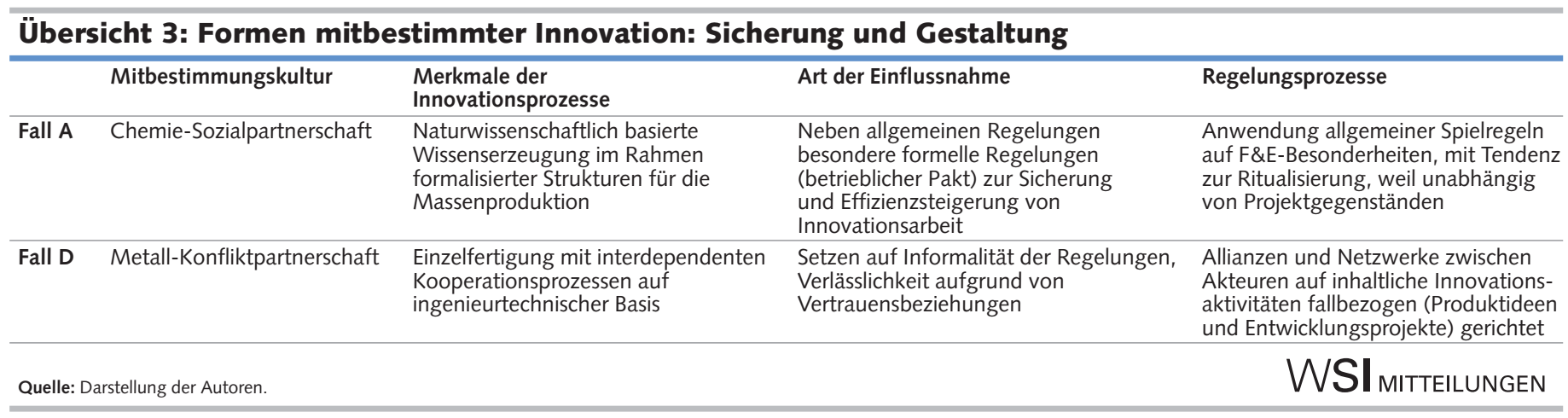

triebsrat im Rahmen seiner Kooperation mit dem Standortmanagement seinen Einfluss zur Sicherung und Verstetigung von F\&E-Prozessen geltend zu machen. Vor dem Hintergrund der einschneidenden Neukonfiguration der betrieblichen Arbeitsbeziehungen sind indes verbindliche Absprachen für den Bereich Innovationsarbeit ausgeblieben, was der Ökonomisierung der Unternehmens- und Betriebsorganisation Raum verschafft, etwa eine stärkere Rigidisierung in der Produktentwicklung mit hohem Zeit- und Ressourcendruck einseitig durchzusetzen, was wiederum die Spielräume der Innovationsarbeiter einschränkt.

Ähnlich stellt sich die Situation in dem Maschinenbauunternehmen $C$ dar. Hier führt der Begründungsdruck, der von den Finanzmarktakteuren auf den hohen Kostenanteil für den Entwicklungssektor ausgeübt wird, zu einer Evaluation des Forschungs- und Entwicklungsbereichs durch ein Beratungsunternehmen. Deren Ergebnisse mündeten ein in ein Organisationsprojekt zur Steigerung der Effizienz und Effektivität des Forschungs- und Entwicklungszentrums, an dessen Ausarbeitung und Implementation die betriebliche Interessenvertretung nicht beteiligt wurde bzw. sich nicht beteiligte. Hier ist es zwar der betrieblichen Interessenvertretung gelungen, in einer Standortsicherung die Höhe des Budgetvolumens für den F\&EBereich festzuschreiben. Ein weiterreichender Anspruch wird jedoch nicht verfolgt.

Eine wesentliche Ursache hierfür ist, dass es in dem Unternehmen eine gewissermaßen gespaltene Mitbestimmungskultur gibt. Das Gravitationszentrum der Interessenvertretung liegt bei den Fertigungsbereichen mit einer facharbeiterspezifischen Vertretungsstärke, während die Innovationsarbeiter und Innovationsarbeiterinnen in der Forschung und Entwicklung kaum eine Repräsentanz im Betriebsrat haben. Trotz praktisch vielfältig genutzter Kooperationsbeziehungen zwischen beiden Bereichen weisen die Arbeitsund Interessenorientierungen von Facharbeitern und Hochqualifizierten immer noch so weit auseinander, dass berufliche und Arbeitsinteressen sehr unterschiedlich artikuliert und zur Geltung gebracht werden. Hierbei erweisen sich die Vertretungsmöglichkeiten der Beschäftigten in Forschung und Entwicklung angesichts einschränkender Verfügungen des Managements etwa hinsichtlich von Arbeitszeitregelungen, die auf Standardisierungen zurückgreifen, durchaus als defizitär. Auch die im Rahmen einer management- und beratungsorientierten Initiative zur Steigerung der Effizienz von Forschung und Entwicklung intendierten Organisationsansätze zielen auf eine stärkere Formalisierung und Standardisierung von Projektarbeit, wobei fraglich bleibt, ob dieser Prozess zu gemeinsam akzeptierten Regelungen der Innovationsarbeit führen kann.

Viele der befragten hochqualifizierten Innovationsarbeiter artikulieren ein dezidiertes Interesse an einer möglichst großen Stabilität des organisatorischen Umfelds, um die Sicherheit zu haben, dass das eigene Innovationshandeln mit seinen Unwägbarkeiten sich zumindest innerhalb möglichst verlässlicher Strukturen und Prozeduren vollzieht. Obwohl entsprechende Sicherungsfunktionen intakter Mitbestimmungsstrukturen in den beiden Fällen $B$ und $\mathrm{C}$ positiv auf die F\&E-Bereiche ausstrahlen und von den Innovationsarbeiterinnen und Innovationsarbeitern anerkannt werden, sind hier allerdings konkrete, bereichsspezifische Gestaltungsimpulse kaum zu erkennen.

\section{2 ...UND GESTALTUNGS- FUNKTIONEN}

Die Formen der Mitbestimmung der Innovation in den beiden anderen untersuchten
Unternehmen kontrastieren diesbezüglich überaus deutlich. In diesen beiden Fällen wirken die Betriebsräte nämlich nicht bloß absichernd „von außen“, sondern auch direkt pro-aktiv gestaltend auf die Spielregeln der Innovation selbst ein. Sie haben nicht nur Sicherungs-, sondern erkennbar auch eine Ermöglichungs- und Gestaltungsfunktion im Hinblick auf Innovation - wenn auch wiederum in recht unterschiedlicher Weise (Übersicht 3).

Im Unternehmen $A$ sind in einem eigenen betrieblichen „Pakt“ - orientiert an der sozialpartnerschaftlichen Tradition eines routiniert-,,professionellen “Umgangs zwischen den Betriebsparteien und nach dem Muster von Standortvereinbarungen für die Produktionsbereiche - gemeinsam verpflichtende Ziele vereinbart worden, um die Effizienz der Forschung zu steigern, Innovationsprozesse zu beschleunigen und Arbeitsplätze in die Zukunft hinein zu sichern. Diese Betriebsvereinbarung enthält zugleich Festschreibungen konkreter und formeller Spielregeln für das Innovationshandeln, vor allem im Hinblick auf die Arbeitszeit- und Leistungsbedingungen, aber auch bezüglich Weiterbildung, Status und Karrierechancen der Laborantinnen und Laboranten. Gerade sie waren es, die bereits früh einen Veränderungsbedarf von Arbeits- und Organisationsstrukturen ermittelt, betriebspolitisch reklamiert und dabei Defizite benannt sowie Lösungsvorschläge erarbeitet hatten, die dann als Forderungen der Interessenvertretung in die Aushandlungen mit dem Management eingeflossen sind.

Hier ist mithin der Regelungsbedarf von Innovationsarbeit als offizielles Aushandlungsfeld der betrieblichen Arbeitsbeziehungen und damit der Mitbestimmung in einem ersten Schritt etabliert. Eine eigene Arena für mitbestimmte Innovation ist sozusagen entstanden, mit der gemeinsamen Verpflichtung zur kontinuierlichen 
Überprüfung der Entwicklung. Dies sichert dem Betriebsrat relativ gute Informationsund Mitwirkungsmöglichkeiten in einem Bereich mit traditionell ungünstigen $\mathrm{Zu}$ gangsbedingungen. Zugleich aber deuten sich auch Risiken einer solchen Strategie an, die unter anderem stark auf formale Regulierung setzt: $\mathrm{Zu}$ beobachten ist eine Tendenz zur Ritualisierung und zur Vervielfältigung von Workshops, Diskussionsveranstaltungen etc., die "neben“ der eigentlichen Innovationsarbeit durchzuführen sind und viel Energie und Motivation verbrauchen.

Sowohl im Politiktyp als auch im Ergebnis unterscheidet sich davon der Fall des Maschinenbauunternehmens $D$, wo von der Unternehmensführung, in der die Eigentümerfamilie aktiv präsent ist, eine „Innovationsoffensive" auf den Weg gebracht wird, die für Innovationsprozesse zusätzliche Ressourcen wie neue Regelungsformen und Zuständigkeiten vorsieht. Seitens des Betriebsrats wird hierbei nicht auf einen formalisierten Abschluss einer betrieblichen Vereinbarung bestanden oder gedrängt. Das resultiert keineswegs aus einem Mangel an Durchsetzungsfähigkeit und Organisationsmacht der betrieblichen Interessenvertretung, die eher im Gegenteil unter anderem mit Konzepten im Rahmen der gewerkschaftlichen Initiative „Besser statt billiger" ihre Gestaltungskraft schon unter Beweis gestellt hat. Vielmehr setzt die betriebliche Interessenvertretung bewusst auf eine gewisse Informalität der Regelungen. Deren hohe Verlässlichkeit kann sichergestellt werden, weil sie auf belastbaren - und konflikterprobten - Vertrauensbeziehungen der betrieblichen Akteure basiert, die eine spezifische Sozialordnung begründen. „Regelungen kommen immer dann zustande, wenn man mit dem Arbeitgeber nicht mehr weiterkommt", lautet hier das Credo des Betriebsrats, der Informationen und Vereinbarungen für den Bereich der Entwicklungsarbeit in informellen, aber gleichwohl verbindlichen Klärungen mit den Protagonisten der Entwicklungsabteilungen vereinbart und sichert. Auf diese Weise werden Allianzen geschmiedet, die auf der Akzeptanz und Wirksamkeit der betrieblichen Mitbestimmung basieren und Spielräume für Innovationen und Innovationsarbeit sicherstellen.

Diese Art mitbestimmter Innovation setzt gewissermaßen mehr auf Inhalte als auf Formen. Thema sind immer wieder vor allem konkrete Produktideen und Ent- wicklungsprojekte, die systematisch auf ihren Nutzen für nachhaltige Beschäftigungssicherung und ihre Auswirkungen auf die Arbeitsbedingungen sowohl der F\&E- als auch der Fertigungs- und Montagebelegschaften hin abgeklopft werden. Starke Persönlichkeiten sowohl im Betriebsrat als auch im Bereichsmanagement tragen diesen Politiktyp. Das aber ist zugleich eine der Schwachstellen eines solchen Mitbestimmungsmusters - schon ein Personenwechsel gefährdet womöglich die langjährig eingespielten informellen Netzwerke und Allianzen, für deren Aufrechterhaltung sozusagen ständige „Beziehungsarbeit" nötig ist.

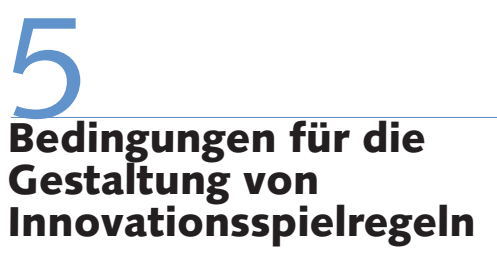

Die dargestellten Fälle belegen, dass eine Mitbestimmung bei Innovationsprozessen möglich und wirksam ist, bei der die Institutionen und Akteure der Arbeitsbeziehungen ihren Part im Spannungsverhältnis von Mitbestimmung und technologischer Innovation spielen. Freilich hat die Einflussnahme der Betriebsräte auch im Rahmen von „good practices“ über die ohnehin bestehenden Machtasymmetrien hinaus ihre Grenzen. Und zugleich bleibt zum einen zu fragen, welche Bedingungen für eine erfolgreiche Mitbestimmung bei Innovation förderlich sind; zum anderen, welche Gründe für die Durchsetzung des einen oder anderen Politiktyps verantwortlich sind.

Was den letzten Punkt anbelangt, so ist in jedem Fall festzuhalten, dass die beiden zuletzt dargestellten, besonders wirksamen Mitbestimmungsmuster sich das eine Mal im Kontext der Mitbestimmungskultur vom Typ „Chemiepartnerschaft“, das andere Mal im Rahmen der Mitbestimmungskultur der Metallindustrie finden. Hier wie da ist nicht nur die Absicherung, sondern auch wirksame Mitgestaltung von Innovationsprozessen möglich. Freilich müssen die Erfolgsbedingungen dafür im Einzelnen noch genauer ausgelotet werden. Sie liegen jedenfalls - wie ja auch zu vermuten war - nicht allein und auch nicht in erster Linie in den Besonderheiten der jeweiligen branchentypischen Vertretungskultur begründet.
Neben unterschiedlichen Ausprägungen betrieblicher Konflikt- und Aushandlungshistorie sowie weiterer Einflussfaktoren scheinen auch Zusammenhänge mit den variierenden Prozessstrukturen und Marktbezügen von Innovationsarbeit eine Rolle zu spielen. Was ist damit gemeint? In Abhängigkeit von Portfolio und stofflichen Erfordernissen finden sich in den Unternehmen unterschiedliche Strukturen der Prozessgestaltung und der sachlichen wie zeitlichen (Ab-)Kopplung von Innovationsaktivitäten. So verläuft der Innovationsprozess im Fall des Chemie-Unternehmens in einem von möglichen späteren fertigungstechnischen bzw. kommerziellen Realisierungen zunächst völlig entkoppelten Verbund von experimenteller Forschung und sogenannter Anwendungstechnik. Dagegen liegt der Schwerpunkt in den anderen Fällen in einer weit verzweigten, deutlich stärker mit Markterfordernissen konfrontierten Produktentwicklung und -konstruktion, die in die fertigungstechnische und kundenbezogene Realisierung der Neuerungen sachlich und zeitlich unmittelbar integriert ist. Diese Prozesse sind zugleich in ganz unterschiedlicher Weise in die Absatzmärkte eingebunden. Hier stehen sich Formen der Programm- oder gar Massenfertigung (wie in den Fällen der Chemie- und Pharmaindustrie und des Maschinenbaus) und Formen kundenspezifischer Einzelfertigung (wie im Fall des Anlagenbaus) gegenüber. Während bei Ersteren die Innovationsprozesse von der direkten Bearbeitung von Kundenaufträgen weitgehend abgeschirmt sind und sozusagen in einem mehr oder weniger geschlossenen eigenen Informations- und Kooperationskreislauf (einschließlich Anwendungstechnik, Prototypenbau etc.) stattfinden, ist die Masse der Entwicklungs- und Konstruktionstätigkeiten bei Einzelfertigung direkt im Rahmen einzelner umfangreicher Kundenaufträge zu leisten, deren Bearbeitung sich oft mehrere Jahre hinzieht. Entsprechend unmittelbar ist Innovationsarbeit in der letztgenannten Konstellation mit den Routinen der Auftragsabwicklung verbunden, insbesondere mit der Fertigung sowie der Inbetriebnahme der Maschinen und Anlagen beim Kunden. Innovation speist sich hier einerseits in hohem Maße aus der oftmals informellen Alltagskooperation mit vielfältigen betrieblichen Akteuren (bis auf die shop-floor-Ebene), sie muss diesem Alltag aber andererseits auch immer wieder mühsam abgerungen werden. 
Die jeweilige „Zugänglichkeit“ von Innovationsprozessen für spezifische Mitbestimmungsstile scheint zumindest auch von Strukturbedingungen der jeweiligen Innovationskonstellation abzuhängen. Bei Einzelfertigung mit vielfach interdependenten Kooperationsprozessen auf ingenieurtechnischer Basis, im teilweise quasi-kleinbetrieblichen Kontext des Maschinenbauund Anlagenbauunternehmens, scheinen sich auch im Hinblick auf die Mitbestimmung beharrlich-verbindliche Informalität und konkreter Sachbezug am ehesten als passend und regelungswirksam zu erweisen. Anders bei naturwissenschaftlich basierter, experimenteller Wissenserzeugung im Rahmen hochgradig formalisierter Strukturen und Innovationen für die Massenproduktion in dem Chemieunternehmen. Hier scheint auch ein eher bürokratischer Interessenvertretungstyp besser geeignet zu sein, um unter solchen Bedingungen die Spielregeln der Innovation erfolgreich mitzubestimmen.

\section{Grenzen der Mitbestim- mung bei Innovationen}

Hinzuweisen bleibt schließlich auf die klaren Grenzen der Mitbestimmung, sobald es um bisherige "Spezialitäten“ der Innovationsbereiche geht. Manche von ihnen bleiben im Windschatten selbst ansonsten erfolgreicher Einflussnahme. Das kann am Beispiel eines bislang eher verborgenen Spielfelds der Innovationsarbeit illustriert werden: „Erfinden“ und „intellectual property“ (IP). Erfindungsmeldungen zu machen bzw. Patentierungen anzustoßen, gehört zur ganz normalen Leistungserwartung der Unternehmen gegenüber Innovationsarbeitern. Nicht wenige der Befragten betreiben Erfindung und Patentierung durchaus als „individuelles Projekt“, von dessen Erfolg auch professionelle Anerkennung abhängt. Und sobald wirklich eine „Erfindung" entsteht, stellt sich stets auch die Frage nach ihrer rechtlichen Aneignung - vor allem durch Patentierung - und nach der Verteilung der sich daraus möglicherweise ergebenden Erträge (Erfindervergütung, Lizenzeinnahmen).

Die Mobilisierung sowie Be- und Verwertung neuen Wissens sind in den Unternehmen offenbar wichtiger geworden. So werden für die unterschiedlichen Produktlinien in den frühen Phasen der Innovationsplanungen generell auch „IP-Strategien" entwickelt. Und es werden organisatorische oder informationstechnische Instrumente eingesetzt bzw. erprobt: sogenannte Ideen-Workshops neben der Projektabwicklung und „Innovationsforen“ als informationstechnischer Raum der Ideenpublikation, -diskussion und -bewertung. Diese Seite des Innovationsgeschehens ist prozedural sehr genau geregelt (durch Arbeitnehmererfindungsgesetz und Patentabteilung), zugleich aber völlig unbeeinflusst von betrieblicher Interessenvertretung. Gleichwohl geht es um Arbeitnehmerinteressen - und es besteht potenziell mitbestimmungsrelevanter Regulierungsbedarf.

Bezogen auf die unmittelbare Innovationsarbeit ermuntern jene Strategien einerseits die erwähnten individuellen „Patentierungsprojekte"; andererseits blenden sie bestimmte Wissensformen und Wissensträger aus. „Erfinden“ findet aber auch eingebettet in eingespielte informelle Regelungen statt, in denen implizite Normen einer gerechten Beteiligung am Erfindungsertrag zum Ausdruck kommen: „Wer kommt auf's Patent?" ist eine immer wieder zu beantwortende Frage. Die Absprachen dokumentieren ein Bewusstsein davon, dass neues Wissen in aller Regel im Kollektiv bzw. im Kontext kollektiver Diskussions- und Arbeitszusammenhänge entsteht.

Dieses Bewusstsein von der kollektiven Natur der Erfindungen hat indes Grenzen. Das zeigt u. a. das Beispiel der Laboranten im untersuchten Chemieunternehmen, die sich benachteiligt und ausgebootet fühlen: Sie werden vom Management und auch von den meisten Laborleitern gar nicht zu dem Kreis jener „Hochqualifizierten“ gezählt, die vom Status her für Erfindungen „zuständig“ sind. Doch auch sie verfügen über ein diesbezüglich überaus relevantes (Erfahrungs-)Wissen, das unter der Hand sehr wohl einfließt und das in der Interessenperspektive dieser Gruppe gerechterweise eben auch offiziell anerkannt werden müsste. Auch hier bestehen also Regelungsbedarfe, die von der betrieblichen und gewerkschaftlichen Interessenvertretung aufzugreifen wären.

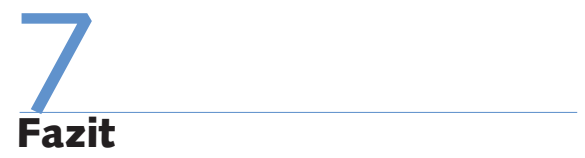

Innovation und Mitbestimmung mögen ein Spannungsfeld sein, aber ein potenziell durchaus produktives. Mitbestimmung erfüllt sowohl Sicherungs-, als auch Gestaltungsfunktionen, die die Bedingungen und die Nachhaltigkeit von Kooperation und Innovativität im Forschungs- und Entwicklungsbereich positiv beeinflussen können. Unsere empirischen Recherchen zeigen, dass der im Hinblick auf Innovationsarbeit bestehende und angesichts von Umstrukturierungen und Ökonomisierungsdruck noch wachsende Regulierungsbedarf durchaus von Institutionen der betrieblichen Mitbestimmung aufgenommen und in der Interessenperspektive der Arbeitnehmer (mit-)gestaltbar ist. Dabei gibt es freilich bedeutsame Differenzen bei der Gewichtung von Sicherungs- und Gestaltungsfunktion und bei den Politikstilen mitbestimmter Innovation.

Einen „one best way" wird man nicht erwarten können, aber die dargestellten Fälle von „good practices“ zeigen zumindest, wie Mitbestimmung durch Absicherung von Innovationsressourcen und durch formalisierte wie informelle Vereinbarungen und Regelungen zur Absorption von Ungewissheit bei Innovationsprozessen beitragen kann. 


\section{LITERATUR}

Blättel-Mink, B. (2006): Kompendium der Innovationsforschung, Wiesbaden

Breschi, S./Malerba, F./Orsenigo, L. (2000): Technological Regimes and Schumpeterian Patterns of Innovation, in: The Economic Journal 463, S. $388-410$

Fagerberg, J. (2005): Innovation: A Guide to the Literature, in: Fagerberg, J./ Mowery, D./Nelson, R. (Hrsg.): The Oxford Handbook of Innovation, Oxford, New York, S. 1-26

Fagerberg, J./Mowery, D./Nelson, R. (Hrsg.) (2005): The Oxford Handbook of Innovation, Oxford, New York
Kädtler, J. (2006): Sozialpartnerschaft im Umbruch. Industrielle Beziehungen unter den Bedingungen von Globalisierung und Finanzmarktkapitalismus, Hamburg

OECD (1995): The Measurement of Scientific and Technological Activities. Proposed Guidelines for Collecting and Interpreting Technological Innovation Data. Oslo Manual, DSTI,OECD/European Commission Eurostat, Paris

Rammert, W. (2008): Technik und Innovation, in: Maurer, A. (Hrsg.): Handbuch der Wirtschaftssoziologie, Wiesbaden, S. 291-319 